\title{
Generating odd-dimensional rotating black holes with equal angular momenta by using the Kerr-Schild Cartesian form of metric
}

\author{
Masoumeh Tavakoli ${ }^{1,2}$, Behrouz Mirza ${ }^{1}$ \\ ${ }^{1}$ Department of Physics, Isfahan University of Technology, Isfahan 84156-83111, Iran. \\ ${ }^{2}$ Research Institute for Astronomy and Astrophysics of Maragha (RIAAM), Maragha, P. O. \\ Box: 55134-441, Iran.
}

\begin{abstract}
The Newman-Janis (NJ) method is a prescription to derive the Kerr space-time from the Schwarzschild metric. The BTZ, Kerr and five-dimensional MyersPerry (MP) black hole solutions have already been generated by different versions of the NJ method. However, it is not known how to generate the metric of higher-dimensional $(d>6)$ rotating black holes by this method.

In this paper, we propose the simplest algorithm for generation of the fivedimensional MP metric with two arbitrary angular momenta by using the KerrSchild form of the metric and quaternions. Then, we present another new twostep version of the NJ approach without using quaternions that generate the five-dimensional MP metric with equal angular momenta. Finally, the extension of the later procedure is explained for the higher odd-dimensional rotating black holes $(d>5)$ with equal angular momenta.

Keywords: Kerr-Schild metric -Higher-dimensional black hole-Quaternion
\end{abstract}

\section{Introduction}

Newman and Janis proposed a procedure for generating rotating metrics (axisymmetric) from static ones (spherically symmetric) in 1965 [1, 2]. The original Newman-Janis (NJ) trick devised for the Kerr metric included four steps as follows: First, inverting the metric to Eddington-Finkelstein coordinates, second, finding a null tetrad basis, then, applying complex conjugate transformations for $r$ and $u$ components and Finally, inverting again the metric Mirza $\left.^{1}\right)$

URL: m.tavakoly@ph.iut.ac.ir (Masoumeh Tavakoli ${ }^{1,2}$ ), b.mirza@iut.ac.ir (Behrouz 
to earlier coordinates followed by changing to the Boyer-Lindquist coordinates. In 1990, another version of the NJ trick was developed by Giampieri, where the null tetrads were avoided, thus facilitate the generalization of the procedure to more complicated cases [3]. Recently, a simple version of the NJ method was proposed in [4, 5], where the authors used both oblate spheroidal and Cartesian coordinates to generate the four-dimensional Kerr-Schild Cartesian form of the Kerr metric.

The five-dimensional Myers-Perry (MP) black hole solution was obtained using simplification of the NJ algorithm proposed by Giampieri [6, 7, 8, 9, 10]. The authors in [10] also proposed a simpler NJ algorithm to generate five-dimensional rotating black holes. An interesting aspect of this approach was the use of quaternions for the first time.

In this paper, we propose the simplest two steps formulation of the NJ method to obtain the five-dimensional MP rotating black holes with two independent angular momenta instead of using the original 4-step NJ prescription. In our approach, we have used both quaternions and the Kerr-Schild form of the metric. However, the extension of this protocol to higher dimensional rotating black holes is not possible. Therefore, we introduce another prescription based on the Kerr-Schild form of the metric to obtain higher dimensional rotating black holes with equal angular momenta.

Recently, an interesting application of the NJ method for calculating self force in four and five-dimensional rotating black holes was proposed in [11, 12].

This paper is organized as follows: In Section 2, we introduce a new approach where the Kerr-Schild form of the five-dimensional Schwarzschild black holes and quaternions are used to generate five-dimensional MP black holes with two angular momenta. In Section 3, we further argue about the distance parameter replacement in our prescription by using rotation and translation of resource in the potential. In Section 4 we introduce another version of the NJ algorithm and the Kerr-Schild form of the metric is generated for higher odd-dimensional rotating black holes with equal angular momenta. In Appendix A, the basic mathematical properties of quaternions are reviewed. In Appendix B, we explain how to generate the Kerr-Schild Cartesian form of the rotating BTZ black hole solution by the simplest version of the NJ method. 


\section{Rotating five-dimensional black holes}

In this Section, we use the Kerr-Schild form of the metric and quaternions to obtain five-dimensional MP black holes, for earlier studies that other versions of the NJ method were introduced see [9] and [10]. In [10], the authors proposed a new algorithm for generating five-dimensional Meyers-Perry black holes using quaternions. This method was simpler to use compared to the earlier proposals in [9].

We introduce a new two-step method to generate five-dimensional black holes. In the Cartesian coordinates, a five-dimensional Schwarzschild metric is as follows:

$d s^{2}=-d \tau^{2}+\sum_{i=1}^{2}\left[d x_{i}^{2}+d y_{i}^{2}\right]+\left(1-f\left(r_{0}\right)\right)\left(\sum_{i=1}^{2}\left[\frac{\left(x_{i} d x_{i}+y_{i} d y_{i}\right)}{r_{0}}\right]+d \tau\right)^{2}$,

where

$$
f\left(r_{0}\right)=1-\frac{m}{r_{0}^{2}}
$$

Therefore, the five-dimensional Schwarzschild geometry in the Kerr-Schild form can be written as follows:

$$
\mathrm{g}_{a b}=\eta_{a b}+\left(1-f\left(r_{0}\right)\right)\left(\ell_{0}\right)_{a}\left(\ell_{0}\right)_{b}
$$

where

$$
\begin{aligned}
\eta_{a b}=\operatorname{diag}\{- & -1,+1,+1,+1,+1\}, \\
\left(\ell_{0}\right)_{a} & =\left(1 ; \hat{r_{0}}\right) \\
= & \left(1 ; \frac{x_{1}}{r_{0}}, \frac{y_{1}}{r_{0}}, \frac{x_{2}}{r_{0}}, \frac{y_{2}}{r_{0}}\right) .
\end{aligned}
$$

We define the following coordinate transformations:

$$
x_{1}+\hat{i} y_{1}=r_{0} \sin \left(\theta_{0}\right) e^{\hat{i} \phi_{01}}, \quad x_{2}+\hat{j} y_{2}=r_{0} \cos \left(\theta_{0}\right) e^{\hat{j} \phi_{02}},
$$

where $\hat{i}$ and $\hat{j}$ are quaternions (Appendix A). Therefore the null co-vector in Eq. (5) can be written as follows:

$$
\left(\ell_{0}\right)_{a}=\left(1 ; \sin \theta_{0} \cos \phi_{01}, \sin \theta_{0} \sin \phi_{01}, \cos \theta_{0} \cos \phi_{02}, \cos \theta_{0} \sin \phi_{02}\right) .
$$

To obtain the MP space-time, we initially use the following oblate spheroidal coordinates $\left(r, \theta, \phi_{1}, \phi_{2}\right)$ :

$$
x_{1}+\hat{i} y_{1}=\left(r+\hat{i} a_{1}\right) \sin \theta e^{\hat{i} \phi_{1}}, \quad x_{2}+\hat{j} y_{2}=\left(r+\hat{j} a_{2}\right) \cos \theta e^{\hat{j} \phi_{2}} .
$$


The null co-vector $\left(\ell_{0}\right)_{a}$ in Eq. (8) can be replaced as follows:

$$
\begin{array}{r}
\left(\ell_{0}\right)_{a} \longrightarrow \ell_{a}=\left(1 ; \sin \theta \cos \phi_{1}, \sin \theta \sin \phi_{1}, \cos \theta \cos \phi_{2}, \cos \theta \sin \phi_{2}\right), \\
=\left(1 ; \operatorname{Re}\left[\frac{x_{1}+\hat{i} y_{1}}{r+\hat{i} a_{1}}\right], \operatorname{Im}\left[\frac{x_{1}+\hat{i} y_{1}}{r+\hat{i} a_{1}}\right], \operatorname{Re}\left[\frac{x_{2}+\hat{j} y_{2}}{r+\hat{j} a_{2}}\right], \operatorname{Im}\left[\frac{x_{2}+\hat{j} y_{2}}{r+\hat{j} a_{2}}\right]\right) \\
=\left(1 ; \frac{x_{1} r+y_{1} a_{1}}{r^{2}+a_{1}^{2}}, \frac{y_{1} r-a_{1} x_{1}}{r^{2}+a_{1}^{2}}, \frac{x_{2} r+y_{2} a_{2}}{r^{2}+a_{2}^{2}}, \frac{y_{2} r-a_{2} x_{2}}{r^{2}+a_{2}^{2}}\right) .
\end{array}
$$

Now, we use a prescription based on quaternions that was introduced in [10]. According to [10], $r$ and $\bar{r}$ are defined in the following equations:

$$
\begin{aligned}
& r=r^{\prime}-\hat{i} a_{1} \cos \theta-\hat{j} a_{2} \sin \theta, \\
& \bar{r}=r^{\prime}+\hat{i} a_{1} \cos \theta+\hat{j} a_{2} \sin \theta .
\end{aligned}
$$

Therefore, we use the following replacement for $1-f\left(r_{0}\right)$ :

$$
\frac{m}{r_{0}^{2}} \longrightarrow \frac{m}{r^{2}}=\frac{m}{r \bar{r}}=\frac{m}{r^{2}+a_{1}^{2} \cos ^{2} \theta+a_{2}^{2} \sin ^{2} \theta} .
$$

It should be noted that quaternions are very useful for this replacement $\left(r_{0}^{2} \rightarrow\right.$ $\left.r^{2}\right)$ in our calculation. Using quaternions leads to a simplest algorithm compared to complex numbers in $[9]$.

Finally, using replacements that defined in Eqs. (10) and (12) in Eq.(3), the defining metric for the MP space-time in the Kerr-Schild Cartesian form is obtained:

$$
\mathrm{g}_{a b}=\eta_{a b}+\frac{m}{r^{2}+a_{1}^{2} \cos ^{2} \theta+a_{2}^{2} \sin ^{2} \theta} \ell_{a} \ell_{b} .
$$

Using Eqs. (10) and (13), we can write the rotating MP metric with two angular momenta in five dimensions in the Cartesian coordinates as follows:

$$
\begin{gathered}
d s^{2}=-d \tau^{2}+\sum_{i=1}^{2}\left[d x_{i}^{2}+d y_{i}^{2}\right]+ \\
(1-f(r))\left(\sum_{i=1}^{2}\left[\frac{r\left(x_{i} d x_{i}+y_{i} d y_{i}\right)-a_{i}\left(x_{i} d y_{i}-y_{i} d x_{i}\right)}{r^{2}+a_{i}^{2}}\right]+d \tau\right)^{2},
\end{gathered}
$$

where

$$
\begin{aligned}
1-f(r) & =\frac{m}{r^{2}+a_{1}^{2} \cos ^{2} \theta+a_{2}^{2} \sin ^{2} \theta} \\
& =\frac{m}{r^{2}+a_{1}^{2}\left(\frac{x_{2}^{2}+y_{2}^{2}}{r^{2}+a_{2}^{2}}\right)+a_{2}^{2}\left(\frac{x_{1}^{2}+y_{1}^{2}}{r^{2}+a_{1}^{2}}\right)} .
\end{aligned}
$$

In this way, by using a simple and quick algorithm, we have obtained the rotating five-dimensional MP metric in the Kerr-Schild coordinates with two angular momenta. In the following Section, the procedure for obtaining Eq. (11) is explained. 


\section{Transformation function using the Kerr-Schild scalar potential:}

It was argued in [4] that in four dimensions, we may explain " $r \rightarrow r-\hat{i} a \cos \theta$ " by a displacement of the potential source of the Schwarzschild solution in the complex plane (displacement of $z$ to $z-\hat{i} a$ ) (see also [13, 14]).

In five dimensions, for obtaining " $r \rightarrow r-\hat{i} a \cos \theta-\hat{j} b \sin \theta$ " we have to rotate the source followed by a move in the complex quaternion plane as follows.

At first, the source is rotated:

$$
\begin{gathered}
x_{i}^{\prime}=x_{i} \sin \phi_{i}-y_{i} \cos \phi_{i}, \\
y_{i}^{\prime}=x_{i} \cos \phi_{i}+y_{i} \sin \phi_{i},
\end{gathered}
$$

where $x_{i}^{\prime 2}+y_{i}^{\prime 2}=x_{i}^{2}+y_{i}^{2}, \quad(i=1,2)$.

Then we replace " $y_{1}^{\prime} \rightarrow y_{1}^{\prime}-\hat{j} b$ " and " $y_{2}^{\prime} \rightarrow y_{2}^{\prime}-\hat{i} a$ ", therefore:

$$
\begin{aligned}
\frac{1}{r_{0}^{2}} \rightarrow \frac{1}{r_{c}^{2}} & =\frac{1}{x_{1}^{\prime 2}+\left(y_{1}^{\prime}-\hat{\jmath} b\right)^{2}+x_{2}^{\prime 2}+\left(y_{2}^{\prime}-\hat{i} a\right)^{2}} \\
& =\frac{1}{x_{1}^{\prime 2}+y_{1}^{\prime 2}-b^{2}-\left(2 y_{1}^{\prime} \hat{\jmath} b\right)+x_{2}^{\prime 2}+y_{2}^{\prime 2}-a^{2}-2\left(y_{2}^{\prime} \hat{i} a\right)} .
\end{aligned}
$$

Using Eq. (9) we have

$$
\begin{array}{ll}
x_{1}^{\prime 2}+y_{1}^{\prime 2}=\left(r^{2}+a^{2}\right) \sin ^{2} \theta, & y_{1}^{\prime}=r \sin \theta, \\
x_{2}^{\prime 2}+y_{2}^{\prime 2}=\left(r^{2}+b^{2}\right) \cos ^{2} \theta, & y_{2}^{\prime}=r \cos \theta,
\end{array}
$$

Replacing Eqs. (18) into Eq. (17), leads to

$$
\begin{gathered}
\frac{1}{r_{c}^{2}}=\frac{1}{\left(r^{2}+a^{2}\right) \sin ^{2} \theta-b^{2}-2 \hat{j} r \sin \theta b+\left(r^{2}+b^{2}\right) \cos ^{2} \theta-a^{2}-2 \hat{i} r \cos \theta a} \\
=\frac{1}{r^{2}-a^{2} \cos ^{2} \theta-2 \hat{j} r \sin \theta b-b^{2} \sin ^{2} \theta-2 \hat{\imath} r \cos \theta a .}
\end{gathered}
$$

Considering the properties of quaternions $(\hat{i} \cdot \hat{j}=\hat{k}$ and $\hat{j} \cdot \hat{i}=-\hat{k})$, Eq. (19) maybe written as:

$$
\frac{1}{r_{c}^{2}}=\frac{1}{(r-\hat{i} a \cos \theta-\hat{j} b \sin \theta)^{2}} .
$$

In this way we have explained " $r \rightarrow r-\hat{i} a \cos \theta-\hat{j} b \sin \theta$ " in five dimensions. It is important to consider the governing rules for $f(r)$ in the Ker-Schild form of metric $\left(g_{a b}=\eta_{a b}+f(r) \ell_{a} \ell_{b}\right)$. In general, for finding $f(r)$, the following rules should be applied [9]:

$$
\begin{array}{r}
r \rightarrow \frac{1}{2}(r+\bar{r})=\operatorname{Rer}, \\
\frac{1}{r} \rightarrow \frac{1}{2}\left(\frac{1}{r}+\frac{1}{\bar{r}}\right)=\frac{\operatorname{Rer}}{|r|^{2}}, \\
r^{2} \rightarrow r \bar{r} .
\end{array}
$$


Therefore, $\frac{m}{r_{0}^{2}}$ can be rewritten as:

$$
\frac{m}{r_{0}^{2}} \rightarrow \frac{m}{r^{2}+a^{2} \cos ^{2} \theta+b^{2} \sin ^{2} \theta} .
$$

As quaternions are not defined in higher dimensions, at this time we cannot extend our arguments to six or higher dimensions. However, for special case of equal angular momenta in odd space-time dimensions, we propose a version of the NJ trick in the following section.

For example in five dimensions by using Eq. (7) and Eq. (9), we have

$$
x_{1}^{2}+y_{1}^{2}+x_{2}^{2}+y_{2}^{2}=r_{0}^{2},
$$

and

$$
x_{1}^{2}+y_{1}^{2}+x_{2}^{2}+y_{2}^{2}=r^{2}+a^{2} .
$$

Comparing Eq. (25) and (26) implies:

$$
r_{0}^{2} \rightarrow r^{2}+a^{2},
$$

It is worth nothing that this replacement works for odd higher dimensions.

In the next Section, we consider the higher-dimensional rotating black holes with equal angular momenta.

\section{Rotating odd-dimensional black holes with equal angular momenta}

Here, we consider the rotating black holes in higher dimensions, where all angular momenta are equal and propose a new version of the NJ algorithm. The focus is on the Kerr-Schild coordinate in the odd space-time dimension.

The $d=2 n+1$ dimensional metric $(d \geq 5)$ in the Cartesian coordinates is as follows:

$$
\begin{aligned}
d s^{2} & =\mathrm{g}_{a b} d x^{a} d x^{b} \\
& =-d \tau^{2}+\sum_{i=1}^{n}\left(d x_{i}^{2}+d y_{i}^{2}\right)+\left(1-f\left(r_{0}\right)\right)\left(\frac{\sum_{i=1}^{n}\left(x_{i} d x_{i}+y_{i} d y_{i}\right)}{r_{0}}+d \tau\right)^{2},
\end{aligned}
$$

where,

$$
f\left(r_{0}\right)=1-\frac{m}{r_{0}^{d-3}} .
$$

The generic form of the Kerr-Schild metric in the d dimensions is presented by [15, 16, 17]:

$$
\mathrm{g}_{a b}=\eta_{a b}+\left(1-f\left(r_{0}\right)\right)\left(\ell_{0}\right)_{a}\left(\ell_{0}\right)_{b},
$$


where, $\eta_{a b}$ is the Minkowski metric and

$$
\begin{aligned}
\left(\ell_{0}\right)_{a}= & \left(1 ; \widehat{r_{0}}\right) \\
& =\left(1 ; \frac{x_{1}}{r_{0}}, \frac{y_{1}}{r_{0}}, \cdots, \frac{x_{n}}{r_{0}}, \frac{y_{n}}{r_{0}}\right) .
\end{aligned}
$$

Consider the following coordinate transformations:

$$
x_{i}=r_{0} \mu_{0 i} \cos \phi_{0 i}, \quad y_{i}=r_{0} \mu_{0 i} \sin \phi_{0 i}, \quad i=1 \ldots n,
$$

or

$$
x_{i}+\hat{i} y_{i}=r_{0} \mu_{0 i} e^{\hat{i} \phi_{0 i}}, \quad \sum_{i=1}^{n} \mu_{0 i}^{2}=1 .
$$

It can be shown that

$$
\sum_{i=1}^{n}\left(x_{i}^{2}+y_{i}^{2}\right)=r_{0}^{2}
$$

In this case, $\left(\ell_{0}\right)_{a}$ can be written as follows:

$$
\left(\ell_{0}\right)_{a}=\left(1 ; \mu_{01} \cos \phi_{01}, \mu_{01} \sin \phi_{01}, \cdots, \mu_{0 n} \cos \phi_{0 n}, \mu_{0 n} \sin \phi_{0 n}\right) .
$$

For obtaining the rotating black holes, we initially introduce the following coordinates

$$
x_{i}+\hat{i} y_{i}=\left(r+\hat{i} a_{i}\right) \mu_{i} e^{\hat{i} \phi_{i}}, \quad \sum_{i=1}^{n} \mu_{i}^{2}=1 .
$$

Therefore, we obtain the following equation:

$$
\sum_{i=1}^{n}\left(x_{i}^{2}+y_{i}^{2}\right)=r^{2}+\sum_{i=1}^{n} a_{i}^{2} \mu_{i}^{2}
$$

It should be noted that the right hand side of Eq. (37) does not depend on $\mu_{i}\left(\sum_{i=1}^{n}\left(x_{i}^{2}+y_{i}^{2}\right)=r^{2}+a^{2}\right)$ for equal angular momenta $\left(a_{i}=a\right)$. However, for even dimensions $(d=2 n+2)$, an unpaired spatial coordinate, $z$, exists $\left(\sum_{i=1}^{n} \mu_{i}^{2}+\alpha^{2}=1, \quad(\alpha=z / r)\right)$. Consequently, this result is not valid for these dimensions with equal angular momenta.

At first step, we replace $\left(\ell_{0}\right)_{a}$ as follows:

$$
\begin{aligned}
\left(\ell_{0}\right)_{a} \longrightarrow \ell_{a}= & \left(1 ; \mu_{1} \cos \phi_{1}, \mu_{1} \sin \phi_{1}, \cdots, \mu_{n} \cos \phi_{n}, \mu_{n} \sin \phi_{n}\right), \\
= & \left(1 ; \operatorname{Re}\left[\frac{x_{1}+\hat{i} y_{1}}{r+\hat{i} a_{1}}\right], \operatorname{Im}\left[\frac{x_{1}+\hat{i} y_{1}}{r+\hat{i} a_{1}}\right], \cdots, R e\left[\frac{x_{n}+\hat{i} y_{n}}{r+\hat{i} a_{2}}\right], \operatorname{Im}\left[\frac{x_{n}+\hat{i} y_{n}}{r+\hat{i} a_{2}}\right]\right) \\
= & \left(1 ; \frac{x_{1} r+y_{1} a_{1}}{r^{2}+a_{1}^{2}}, \frac{y_{1} r-a_{1} x_{1}}{r^{2}+a_{1}^{2}}, \cdots, \frac{x_{n} r+y_{n} a_{n}}{r^{2}+a_{n}^{2}}, \frac{y_{n} r-a_{n} x_{n}}{r^{2}+a_{n}^{2}}\right) .
\end{aligned}
$$


At the second step, for equal angular momenta, comparing Eqs. (34) and (37) implies the following replacement:

$$
r_{0}^{2} \rightarrow r^{2}+a^{2}
$$

leading to the following transformation $\left(1-f\left(r_{0}\right) \rightarrow 1-f(r)\right)$ :

$$
\frac{m}{\left(r_{0}^{2}\right)^{\frac{d-3}{2}}} \rightarrow \frac{m}{\left(r^{2}+a^{2}\right)^{\frac{d-3}{2}}} .
$$

Finally, the following metric is obtained for the rotating black holes

$$
\mathrm{g}_{a b}=\eta_{a b}+(1-f(r))(\ell)_{a}(\ell)_{b} .
$$

Hence for equal angular momenta $a_{i}=a$, we have:

$$
\begin{aligned}
d s^{2} & =-d \tau^{2}+\sum_{i=1}^{n}\left(d x_{i}^{2}+d y_{i}^{2}\right)+ \\
& (1-f(r))\left(\sum_{i=1}^{n} \frac{r\left(x_{i} d x_{i}+y_{i} d y_{i}\right)-a\left(x_{i} d y_{i}-y_{i} d x_{i}\right)}{r^{2}+a^{2}}+d \tau\right)^{2},
\end{aligned}
$$

where

$$
f(r)=\frac{m}{\left(r^{2}+a^{2}\right)^{\frac{d-3}{2}}} .
$$

In this way, by using the Kerr-Schild coordinates, the metric of rotating $(2 \mathrm{n}+1)$ dimensional black holes with equal angular momenta is obtained by employing a simple algorithm.

\section{Conclusion}

In this paper, we proposed a simple approach for deriving rotating black holes from static ones. The Kerr-Schild coordinates and the quaternion features are used in this approach for obtaining five-dimensional rotating black holes. This algorithm includes two steps, i.e. replacement of the null co-vectors in the KerrSchild coordinates $\left(\ell_{0}\right)_{a} \rightarrow \ell_{a}$ and use of quaternions for replacing $f\left(r_{0}\right)$ by $f(r)$. We also explain the process of obtaining the distance parameter replacement by using rotation and translation of resource in the potential. At this time we cannot generalize our five dimensional approach to higher dimensions, and it remains for future studies. In the second part of our paper, we proposed a new version of the NJ algorithm for higher odd-dimensional black holes with equal angular momenta for the first time. 
For the future works, it is interesting to generalize the method proposed in this paper to obtain metrics of rotating black holes with unequal angular momenta and also other tips of black holes.

\section{Acknowledgements}

This work has been supported financially by Research Institute for Astronomy and Astro physics of Maragha (RIAAM) under research project No.1/575050.

\section{Appendix A}

Numerical system of quaternions was used to extend complex numbers. Quaternions are generally represented as follows:

$$
A=a_{1}+\hat{i} a_{2}+\hat{j} a_{3}+\hat{k} a_{4}
$$

Furthermore, they can be defined by introducing the elements of a Hamilton basis $\hat{i}, \hat{j}, \hat{k}$, satisfying the following rules:

$$
\begin{aligned}
& \hat{i}^{2}=\hat{j}^{2}=\hat{k}^{2}=\hat{i} \cdot \hat{j} \cdot \hat{k}=-1, \\
& \hat{i} \cdot \hat{j}=\hat{k}, \quad \hat{j} \cdot \hat{i}=-\hat{k} .
\end{aligned}
$$

The conjugate of $A$ is defined as below:

$$
\bar{A}=a_{1}-\hat{i} a_{2}-\hat{j} a_{3}-\hat{k} a_{4}
$$

and its norm is defined as follows:

$$
|A|^{2}=A \bar{A}=a_{1}^{2}+a_{2}^{2}+a_{3}^{2}+a_{4}^{2} .
$$

We use this quantity to obtain (12).

\section{Appendix B}

The Newman-Janis method can be used to obtain rotating BTZ black holes [18, 19]. In [20], four-dimensional transformations were used to derive rotating BTZ black hole solution from the non-rotating one, and the final threedimensional result was presented in the equatorial plane (slice in $\theta=\frac{\pi}{2}$ ). We propose a simple trick to obtain three-dimensional rotating BTZ black holes. 
This simple method is based on the Kerr-Schild coordinate system.

In Cartesian coordinates, the non-rotating(static) three-dimensional BTZ metric is as follows:

$$
\begin{aligned}
d s^{2} & =\mathrm{g}_{a b} d x^{a} d x^{b} \\
& =-d \tau^{2}+d x_{0}^{2}+d y_{0}^{2}+\left(1-f\left(r_{0}\right)\right)\left(\frac{x_{0} d x_{0}+y_{0} d y_{0}}{r_{0}}+d \tau\right)^{2},
\end{aligned}
$$

where

$$
f\left(r_{0}\right)=\left(-M+r_{0}^{2} / \ell^{2}\right) .
$$

The components of metric in (49) can be written as:

$$
\mathrm{g}_{a b}=\eta_{a b}+\left(1-f\left(r_{0}\right)\right)\left(\ell_{0}\right)_{a}\left(\ell_{0}\right)_{b} ;
$$

where

$$
\begin{aligned}
\eta_{a b}= & \operatorname{diag}\{-1,+1,+1\}, \\
\left(\ell_{0}\right)_{a}=\left(1 ; \hat{r_{0}}\right) & \\
= & \left(1 ; \frac{x_{0}}{r_{0}}, \frac{y_{0}}{r_{0}}\right) .
\end{aligned}
$$

Using the following coordinate transformation

$$
x+\hat{i} y=r_{0} e^{\hat{i} \phi_{0}},
$$

a new form of $\left(\ell_{0}\right)_{a}$ can be obtained as follows:

$$
\left(\ell_{0}\right)_{a}=\left(1 ; \cos \phi_{0}, \sin \phi_{0}\right) .
$$

To derive rotating space-time black holes from static one, the following coordinates are initially used

$$
x+\hat{i} y=(r+\hat{i} a) e^{\hat{i} \phi},
$$

where $a$ is the angular momentum parameter and transforms $\left(\ell_{0}\right)_{a}$ to $\ell_{a}$ as follows:

$$
\begin{aligned}
\left(\ell_{0}\right)_{a} \longrightarrow \ell_{a} & =(1 ; \cos \phi, \sin \phi) \\
& =\left(1 ; \operatorname{Re}\left[\frac{\mathrm{x}+\hat{i} y}{r+\hat{i} a}\right], \operatorname{Im}\left[\frac{x+\hat{i} y}{r+\hat{i} a}\right]\right) \\
& =\left(1 ; \frac{r x+a y}{r^{2}+a^{2}}, \frac{r y-a x}{r^{2}+a^{2}}\right) .
\end{aligned}
$$

Next, we replace

$$
\left(-M+r_{0}^{2} / \ell^{2}\right) \longrightarrow\left(-M+r^{2} / \ell^{2}\right),
$$


the following transformation is used, $r_{0} \longrightarrow r+\hat{i} a \sqrt{1-\mu^{2}}$ where $\mu$ is equal to one for BTZ black holes. Finally, we have

$$
\mathrm{g}_{a b}=\eta_{a b}+\left(1-\left(-M+r^{2} / \ell^{2}\right)\right)(\ell)_{a}(\ell)_{b} .
$$

That is, the rotating three-dimensional BTZ metric may be obtained as follows:

$$
\begin{gathered}
d s^{2}=-d \tau^{2}+d x^{2}+d y^{2}+(1-f(r))\left(\frac{r(x d x+y d y)-a(x d y-y d x)}{r^{2}+a^{2}}+d \tau\right)^{2}, \\
f(r)=\left(-M+r^{2} / \ell^{2}\right) .
\end{gathered}
$$

\section{References}

\section{References}

[1] E. T. Newman and A. I. Janis, "Note on the Kerr Spinning-Particle Metric”, Journal of Mathematical Physics 6.6. (June 1965), pp. 915917.

doi: 10.1063/1.1704350.

[2] E. T. Newman, E. Couch, K. Chinnapared, A. Exton, A. Prakash and R. Torrence, "Metric of a Rotating, Charged Mass", Journal of Mathematical Physics 6.6. (June 1965), pp. 918919.

doi:10.1063/1.1704351.

[3] Giacomo Giampieri, "Introducing angular momentum into a black hole using complex variables", Gravity Research Foundation essay contest, (1990). gravityresearchfoundation.org/pdf/awarded/1990/giampieri.pdf

[4] Del Rajan and M. Visser, "Cartesian Kerr-Schild variation on the Newman-Janis trick", 14 Feb 2017; arXiv:1601.03532v2 [gr-qc]. doi:10.1142/S021827181750167X

[5] M. Visser, "The Kerr spacetime: A brief introduction", Cambridge University Press, Cambridge, 2009; arXiv:0706.0622 [gr-qc].

Published in The Kerr spacetime: Rotating black holes in general relativity,

edited by David Wiltshire, Matt Visser, and Susan Scott.

[6] H. Erbin, "Deciphering and generalizing Demianski-Janis-Newman algorithm", Gen. Rel. Grav. 48 (2016) 56; arXiv:1411.2909 [gr-qc]. doi:10.1007/s10714-016-2054-1. 
[7] H. Erbin and L. Heurtier, "Supergravity, complex parameters and the Janis-Newman algorithm", Class. Quant. Grav. 32 (2015) 165005; arXiv:1501.02188 [hep-th]. doi:10.1088/0264-9381/32/16/165005.

[8] H. Erbin, "Janis-Newman algorithm: Generating rotating and NUT charged black holes", Universe 2017, 3(1), 19; arXiv:1701.00037 [gr-qc]. doi:10.3390/universe3010019.

[9] H. Erbin and L. Heurtier, "Five-dimensional Janis-Newman algorithm", Class. Quant. Grav. 32 (2015) 16, 165004; arXiv:1411.2030 [gr-qc]. doi:10.1088/0264-9381/32/16/165004.

[10] Zahra Mirzaiyan, Behrouz Mirza, and Elham sharifian, "Generating five dimensional Myers-Perry black hole solution using Quaternions", Annals of Physics 389 (2018) 11-18; arXiv: 1708.08969[gr-qc]. doi:10.1016/j.aop.2017.12.005.

[11] Matteo Broccoli, Adriano Vigano, "Electromagnetic self-force in curved spacetime: New insights from the Janis-Newman algorithm", Phys. Rev. D 98, 084007 (2018); arXiv:1807.08313 [gr-qc]. doi:10.1103/PhysRevD.98.084007.

[12] Hamideh Nadi, Behrouz Mirza, Zahra Mirzaiyan, "Electromagnetic selfforce in the five dimensional Myers-Perry space time", Annals of Physics 406 (2019) 142-151; arXiv:1904.06553 [gr-qc]. doi:10.1016/j.aop.2019.04.008.

[13] Nilton O. Santos,, "Newtonian Potential and the Complex Space", Lett. al Nuovo Cimento 14 (1975) 327-329.

[14] Menahem M. Schiffer, Ronald J. Adler, James Mark, and Charles Sheffield, "Kerr geometry as complexified Schwarzschild geometry", Journal of Mathematical Physics 14 (1973) 52-56. http://dx.doi.org/10.1063/1.1666171.

[15] Leong Chung Wei Bernard, "Black Holes in Higher Dimensions", http://citeseerx.ist.psu.edu/viewdoc/similar?doi=10.1.1.44.9479.

[16] G.W. Gibbons, H. Lu , Don N. Page and C.N. Pope, "The General Kerr-de Sitter Metrics in All Dimensions", J. Geom. Phys. 53:49-73,2005; 
arXiv:hep-th/0404008v3.

doi:10.1016/j.geomphys.2004.05.001.

[17] Muraari Vasudevan, Kory A. Stevens and Don N. Page, "Separability of the Hamilton-Jacobi and Klein-Gordon Equations in Kerr-de Sitter Metrics", Class. Quant. Grav. 22 (2005) 339-352; arXiv:gr-qc/0405125v2.

doi:10.1088/0264-9381/22/2/007.

[18] Maximo Banados, Claudio Teitelboim, Jorge Zanelli, "The Black Hole in Three Dimensional Space Time", Phys.Rev.Lett. 69 (1992) 1849-1851; arXiv:hep-th/9204099. doi:10.1103/PhysRevLett.69.1849.

[19] Maximo Banados, Marc Henneaux, Claudio Teitelboim, Jorge Zanelli, "Geometry of the 2+1 Black Hole", Phys.Rev.D 48:1506-1525,1993; arXiv:gr-qc/9302012. doi:10.1103/PhysRevD.48.1506.

[20] Hongsu Kim, "Spinning BTZ Black Hole versus Kerr Black Hole : A Closer Look", Phys. Rev. D 59 (1999) 064002; arXiv:gr-qc/9809047v2. 